\title{
Study on Systematic Modeling Method of Fractional Calculus

\author{
Lili $\mathrm{Xu}^{1, \mathrm{a}}$ \\ aemail jxdxxll@163.com
} \\ ${ }^{1}$ Heilongjiang University of Technology, Jixi, Heilongjiang, China, 158100
}

Keywords: Study, Systematic Modeling Method, Fractional Calculus

\begin{abstract}
In this paper, we introduce the definition of fractional calculus and prove the existence and uniqueness of the solution of fractional linear ordinary differential equation by using Laplace transform method, and give the description of transfer function and state equation. Fractional linearity and the simulation results of a viscoelastic system are given to illustrate the effectiveness of the method.
\end{abstract}

\section{Introduction}

The emergence of fractional calculus has been more than 300 years of history. Almost the concept of classical calculus has just been put forward, fractional calculus by many scholars of all ages. The earliest systematic study of fractional calculus was done by Liouville (1832), Riemann (1853) and Holmgren (1864) in the early and middle of the 19th century, but Grünwald and Krug first united the definitions of Liouville and Riemann fractional calculus. The theory and application of fractional calculus in the twentieth century has been remarkably developed. Weyl, Hardy, Littlewood, Kober, Osler, Kuttner, Riesz, Erdélyi, Higgis, M. Caputo and Miller-Ross all made important contributions.

The application of fractional calculus is very extensive. It includes some scientific and engineering applications: memory, mechanical and electrical characterization of various materials, rheological characterization of rocks, seismic analysis, viscoelastic damper, power fractal network, Fractional-order sinusoidal oscillators, robotics, electronic circuits, electrolytic chemistry, fractional capacitance theory, electrode-electrolyte interface description, fractal theory, in particular, describing dynamic processes of self-similar and porous structures; fractional-order PID controller design; viscoelastic systems; and Vibration control of softly constructed objects, fractional order of biological neurons and probability theory.

Fractional calculus has been used for more than 300 years. It is widely used in the fields of memory, mechanics and electrical characterization of various materials, rheological characterization of rocks, seismic analysis, viscoelastic damper, power fractal network Fractional-order sinusoidal oscillators, robotics, electronic circuits, electrolytic chemistry, fractional capacitance theory, electrode-electrolyte interface description, fractal theory, in particular, describe dynamic processes of self-similar and porous structures, fractional PID controller design, vibration control of viscoelastic systems and soft structures, fractional order of biological neurons and probability theory, etc. In this paper, two methods are used to analyze the existence and uniqueness of solutions of fractional linear equations and the solution formula is given in order to apply the fractional calculus to the modeling of the actual system.

\section{The Related Research of Fractional Calculus}

The founding of fractional calculus can be traced back to the time when ordinary calculus was established. Leibniz studied the fractional derivative and many other famous mathematicians have studied fractional calculus, and fractional calculus to improve and make an important contribution to the development. Fractional calculus is the study and application of any order (real and complex) differential and integral disciplines, involving functional theory, differential equations, integral equations and mathematical analysis of many other contents. In many disciplines of development and stimulation, the theory of fractional calculus is also growing and in many areas has been 
applied, especially in chemistry, materials science and mechanics has a wide range of applications.

\section{The Definition of Fractional Calculus}

Fractional calculus is essentially non-integer order calculus or arbitrary order calculus and the order can be real or even complex. Mathematicians from different perspectives gave the different definitions of fractional calculus and the definition of rationality has been tested in practice.

The Definition of Grunwald-Letnikov. The fractional calculus defined by (G-L) is such that for any real number $\alpha$, the integer part of $\alpha$ is $([\alpha]$, (that is, $[\alpha]$ is the largest integer less than $\alpha$ ). In the interval $[\alpha, \mathrm{t}]$, there are $\mathrm{m}+1$ order derivative and then the definition of fractional order $\alpha$ differential is:

$$
{ }_{\alpha} D_{\mathrm{t}}^{\alpha} f(t)=\lim _{h \rightarrow 0} h^{-\alpha} \sum_{j=0}^{|(t-\alpha) / h|}(-1)\left(\frac{\alpha}{j}\right) f(t-j h)
$$

The Definition of Riemann-Liouville. Let $\mathrm{T}$ be a positive real number; $\mathrm{L}_{1}[0, \mathrm{~T}]$ denote the set of absolute real functions on $[0, T]$. The set of constructed function $f(x) \in L_{1}([0, T])$. By using Fubini theorem (exchange integral order), it is easy to prove.

$$
\int_{0}^{x} d x \int_{0}^{x} d x \ldots \int_{0}^{x} f(x) d x=\frac{1}{\Gamma(n)} \int_{0}^{x}(x-t)^{n-1} f(t) d t
$$

Caputo Definition. For any $\alpha>0$, the integer part of $\alpha$ is $[\alpha]$, (that is, $[\alpha]$ is the largest integer less than $\alpha$ ). Suppose the function $\mathrm{f}(\mathrm{t})$ has $\mathrm{m} 1+$ Derivative, then the fractional order $\alpha$ is defined as:

$$
{ }_{\alpha} D_{\mathrm{t}}^{\alpha} f(t)=\frac{1}{\Gamma(m-\alpha)} \int_{0}^{t} \frac{f^{m}(\tau)}{(t-\tau)^{\alpha-m+1}} d t
$$

It can be seen from the above analysis that the fractional integral is not only closely related to the value near the present moment but also to the whole history, The time of planning the relevant materials should be, so as to be more widely used. However, because it is a Volterra type integral, in the calculation of long-time numerical integration, for example, to solve the integral-differential equation with fractional derivative or fractional integral, the conventional calculation method will encounter a series of problems: Numerical calculation requires the storage of the entire historical data, with the increase of time, the amount of information increases, the calculation of the rapid growth in workload, and the second is when the time increases, the dispersion error is difficult to control, which may lead to the calculation of data distortion Therefore, the conventional discrete method is not applicable to the effective discretization method of the numerical simulation of material and structural long time mechanical behavior described by fractional derivative.

\section{The Numerical Calculation Method of Fractional Order Differential Operator}

Fractional order system is a nonlinear system and the fractional order control system research must adopt some special methods. The fractional order control system rational function approximation, discretization of fractional order control system is one of the main tools. In this way, the fractional-order control system can be transformed into a general control system to study. At present, the fractional order control system approximation of rational function, discrete mainly using continuous fraction expansion (CFE) method to achieve, even the fraction expansion has many advantages: exponential expansion than the convergence of faster, and can be applied to the frequency domain medium.

The fractional expansion (CFE) method cannot guarantee that the discretization model can keep the stability of the original fractional model and is not ideal in the realization precision. Therefore, the discretization process can also adopt the stable rational function fitting method achieve. The 
optimal rational function fitting method can overcome the shortcomings of CFE method, and the fitting effect is better than continuous fraction method. A. Charef who put forward their own methods has achieved good results. The following is a brief introduction to the Carlson method with continuous fraction approximation and the method with the best rational function fitting. This paper mainly uses the method of complete the numerical calculation.

\section{The Fractional Order Control System Analysis}

For a fractional order system where computational complexity is usually very high, MATLAB undoubtedly takes advantage of the ability to create a class of fractional-order transfer functions, in which functions such as addition, subtraction, multiplication, and feedback of fractional-order transfer functions are written, And overload the required control system analysis function, so that you can like the integer order transfer function that can not only be described in MATLAB can also be time domain and frequency domain analysis.

Time Domain Analysis. It can be seen that the analytic solution of the fractional differential equation is very complex and unfavorable to the engineering realization. Therefore, the numerical solution is generally used when analyzing the fractional calculus equation. In order to facilitate the analysis, we simplify the differential equation:

$$
a_{n} D^{a_{n}} y(t)+\cdots a_{1} D^{a_{1}}+a_{0} D^{a_{0}} y(t)=u(t)
$$

Based on the above formula can write an overload function to achieve arbitrary input fractional system of numerical solution. Based on this function, you can then write a step response of the overloaded function on the e / 76) to draw the fractional system step response curve, just set the unit step function u can be a. Consider the transfer function of a typical integer-order system as follows:

$$
G(s)=\frac{1}{2 s^{2}+3 s+1}
$$

Frequency Domain Analysis. For the fractional-order transfer function, the transfer function can be converted to function. In the transfer function and this is expressed as a combination of real and imaginary parts So that the amplitude and phase of the fractional system can be obtained, and the frequency domain response data of the system can be obtained. According to the obtained frequency response data, we can draw the overloaded function of Bode, Nyquist and Nichols.

It can be seen from the above figure that the slope of the Bode diagram of a fractional system is no longer limited to an integral multiple of $20 \mathrm{~dB} / \mathrm{dec}$ compared to the slope of the Bode diagram of a conventional integer-order system. This characteristic is very good for improving the performance of the control loop So that the design of the transfer function of the correction loop can be freely selected according to the need, for example, by introducing a low-order differential effect near the cut-off frequency to set a smooth slope to enhance the robustness of the system.

The Steady-State Error. The steady-state error parameters of fractional systems are similar to those of integer order systems.

Position error parameter:

$$
\lim _{s \rightarrow 0} G(s)
$$

Speed error parameter:

$$
K_{v}=\lim _{s \rightarrow 0} s G(s)
$$

Acceleration error parameter:

$$
K_{a}=\lim _{s \rightarrow 0} s^{2} G(s)
$$

Steady-state error of the fractional-order system's steady-state error parameters is similar to integer-order systems. 


\section{The Simulation Examples}

A viscoelastic system can be expressed as a fractional differential equation

$$
m D^{2} x(t)+c D^{\alpha} x(t)+k x(t)=u(t)
$$

$\mathrm{DAx}(\mathrm{t})$ is the A-order Caputo derivative $(0<\mathrm{A}<1)$ of the displacement function $\mathrm{x}(\mathrm{t})$, where $\mathrm{m}=$ 1 and $\mathrm{c}=0.5$, where $\mathrm{m}, \mathrm{c}$ and $\mathrm{k}$ are mass, damping coefficient and elastic coefficient respectively. is described as follows: $\mathrm{k}=1, \mathrm{~A}=0.5$, $\mathrm{a} 1=0, \mathrm{a} 2=1$, $\mathrm{u}(\mathrm{t})$ is the input signal,

Using the fractional order equation to solve the equation, the equation of the state equation is given by the following formula: (1) The Laplace transform method is used directly. It is two orders of magnitude faster than that of method, and the solution formula of method is simple and clear, and the state variables can be obtained at the same time, the disadvantage is that when the number of state variables is very large, the solution is not easy. The method is not affected by this, but the calculation time is greatly prolonged when the fractional derivative term is large.

\section{Conclusion}

Fractional calculus is an extension of integer calculus, which has many different characteristics from integer calculus, especially fractional calculus has long-term memory function, especially suitable for the establishment of some mathematical modeling that materials mechanical and electrical properties and a variety of other engineering applications. The fractional calculus for engineering modeling will be a new research direction.

\section{References}

[1] Wang Zhenbin. Journal of Shanghai Jiaotong University, Vol. 6 (2014) No 53, p.25-26

[2] Cao Guangyi, Wang Yunhui. Renewable and Sustainable Energy Reviews, Vol. 12 (2015) No 27, p.74-76

[3] Qian Xiyuan, Jing Jianfen, Hou XuSiem. Electrochem, Vol. 30 (2014) No 19, p.144-145

[4] Wang Kuailiang. Mining Machinery, Vol. 29 (2008) No 27, p.21-23

[5] Zhang Gongxu, Sun Jing. Signal Processing, Vol. 8 (2013) No 27, p.57-60

\section{Lili Xu Female Jixi of Heilongjiang province 198007 Bachelor degree lecturer}

